\title{
KATERINA REED-TSOCHA
}

\section{[}

○ estúdio como estudo: reflexões

sobre a implantação de programas

de doutorado em belas-artes

\section{Tradução: Denise Spier}

\section{RESUMO}

Este artigo vem juntar-se ao debate sobre a natureza exata do doutorado em arte, uma qualificação já consolidada em alguns países, mas um empreendimento ainda recente entre escolas de arte do Reino Unido e dos Estados Unidos. Tomando como ponto de partida a experiência da implantação e desenvolvimento do programa de doutorado da Escola Ruskin de Desenho e Belas-Artes da Universidade de Oxford, proponho questões sobre a razão fundamental da introdução desse grau de pesquisa, a integração de programas de pós-graduação em arte dentro do ambiente de pesquisa de grandes instituições acadêmicas, bem como a orientação e aspectos de projetos de doutorado. Outra questão levantada é o perigo de perda da autonomia e a excessiva academização da prática artística, e sua potencial deterioração em ilustração de ideias teóricas.

\section{PALAVRAS-CHAVE}

Arte e pesquisa. Doutorado em belas-artes. Escola de arte. Programas de doutorado. 


\section{O ESTÚDIO COMO ESTUDO: REFLEXÕES SOBRE A IMPLANTAÇÃO DE PROGRAMAS DE DOUTORADO EM BELAS-ARTES}

Neste artigo optou-se por traduzir fine art pela designação belas-artes, mesmo estando com seu uso em declínio no português, atendendo as proposições do texto; a tradução também poderia ser apenas artes ou arte, dependendo do contexto de cada ocorrência. Practice-led PhD e Studio PhD são sinônimos, significando doutorado com base prática. MFA é sigla em inglês para Master of Fine Arts, mestre (ou mestrado) em belas-artes, grau com foco na criação, incluindo artes visuais, cinema, escrita criativa, teatro, dança e outras artes performáticas. Difere de MA, Master of Arts, mestre em artes, que possui foco essencialmente teórico, envolvendo disciplinas de artes, humanidades, ciências sociais etc. Para MFA e MA as circunstâncias de participação e os requisitos de obtenção de grau podem variar de pais para pais e de instituição para instituição. DPhil é sigla literal para doutor em filosofia, Doctor of Philosophy, o mesmo que $\mathrm{PhD}$, doutor. N. do E.

0 que pode surgir como tendência da língua inglesa - que meramente reflete a grande familiaridade $\mathrm{e}$ envolvimento do autor com esse segmento específico de debates sobre 0 doutorado em arte - tomara venha a servir como um convite para enriquecer 0 debate a partir de novas perspectivas, vindas da experiência de programas como os em andamento no Brasil ou na França.
O Doutorado em Belas-Artes,* conhecido no mundo da língua inglesa' como Practice-led PhD ou Studio $\mathrm{PhD}^{2}$ [sinônimo da anterior], é novo para alguns de nós e bem consolidado em outros lugares. $\mathrm{Na}$ medida em que mais escolas de arte estão aderindo a esse empreendimento (por vezes sucumbindo a pressões institucionais para aliar-se com estruturas existentes através de outros departamentos da universidade e para produzir "pesquisa" para fins de avaliação, como, por exemplo, aquelas exercidas por diversas reencarnações do Research Assessment Exercise [exercício de avaliação das atividades de pesquisa] no Reino Unido), enquanto outras consideram a sua introdução, o debate sobre a sua natureza exata tem, novamente, adquirido impulso. Isso se reflete na proliferação de publicações sobre o tema. ${ }^{3} \mathrm{Em}$ um recente volume intitulado Artists with PhDs: On the new Doctoral Degree in Studio Art [ou Artistas com doutorado: sobre o novo grau doutoral em prática artística], James Elkins, um franco defensor da alegação de que o termo pesquisa é inaplicável à prática artística, ${ }^{4}$ reuniu uma série de ensaios teóricos sobre o novo doutorado, incluindo propostas para diferentes tipos de tais programas, ${ }^{5}$ juntamente com estudos de caso que analisam projetos específicos de doutorado em curso na Austrália e no Reino Unido. As conclusões são, no mínimo, razoavelmente animadoras. Escrevendo previamente à implantação do programa de pós-graduação em sua própria instituição de origem - a School of the Art Institute, em Chicago, onde o programa veio complementar o consagrado programa de mestrado em belas-artes, MFA - , Elkins conclui ensaiando algumas objeções possíveis em uma passagem que vale a pena transcrever na íntegra:

$$
\begin{aligned}
& \text { E, além disso - você poderá dizer - quantos alunos de MFA } \\
& \text { estão aptos para uma pesquisa séria? Ou, se você não aprova } \\
& \text { a palavra "pesquisa", então como explica que tantos podem } \\
& \text { escrever } 50.000 \text { ou } 100.000 \text { palavras sobre qualquer assunto? E } \\
& \text { veja o que aconteceu no Reino Unido: algumas instituições que } \\
& \text { concedem doutorado são interessantes, mas outras são uma } \\
& \text { espécie de mestrado prolongado, com os alunos apenas sentados } \\
& \text { em seus estúdios por outros dois ou três anos, produzindo }
\end{aligned}
$$


mais da mesma arte, olhando para o próprio umbigo, tentando alcançar um pináculo do autoconhecimento que pode ou não tornar seu trabalho mais interessante. ${ }^{6}$

No Reino Unido, uma das mais antigas e frequentemente citadas conceituações da possibilidade de arte como pesquisa em um contexto acadêmico remonta a 1993, quando Christopher Frayling, reitor do Royal College of Art, ensaiou alguns pensamentos iniciais em um breve artigo intitulado "Pesquisa em Arte e Design", reivindicando que a pesquisa "tem sido, pode ser e continuará a ser um importante, talvez o mais importante, alimento para a prática e o ensino da arte, artesanato e design". ${ }^{7}$ Do lado oposto do espectro teórico, no entanto, a aplicabilidade do termo "pesquisa" para a prática artística é duramente contestada. Um forte exemplo pode ser encontrado em um artigo de Charles Harrison, de pouco mais de uma década atrás, sob o sugestivo título "When management speaks" (derivado de um slogan cunhado pelo grupo de arte conceitual Art \& Language). Traçando as origens desta terminologia nas estruturas introduzidas pela avaliação de pesquisa institucional, e alegando com veemência que estes processos de avaliação e as formas de autoinstitucionalização que exigem são incompatíveis com os princípios da pesquisa, Harrison descreve a "embaraçosa e triste" situação oriunda como um resultado da confluência de interesses entre

[...] aqueles que estão ansiosos, por razões políticas, em apoiar a assim chamada pesquisa em arte, e aqueles artistas contratados como professores de ensino superior - se é que podem ser dedicados, mesmo enganados ou trapaceados e sem perspectivas - que precisam defender a credibilidade do crescimento de fundos de seus departamentos. ${ }^{8}$

Harrison, referindo-se a "mistificadores projetos de pesquisa" e a questionável reconceitualização da teoria como pesquisa, escreveu: "A ironia dessa situação é que aqueles que defenderam a importância crítica da Teoria da Arte trinta anos atrás, o fizeram em clara oposição a mistificatória equação da prática artística mais pesquisa. A teoria é, penso, de algum mérito nas artes como crítica e como uma forma de insurgência - e é melhor ficar longe de entidades de financiamento". ${ }^{9}$ E continua:

Também pode ser verdade que a proliferação da Teoria da Arte como uma disciplina quasi-academic irá, afinal, oferecer algumas oportunidades devido ao rigoroso e geralmente muito tedioso estudo. No entanto, há que ser dito que a maior parte
Decisões relativas à escolha de termos estão repletas de dificuldades. Practice-led visa focar a primazia da prática artistica, como também parece envolver o pressuposto implicito de que a prática "precede" de alguma forma pouco clara qualquer investigação teórica, e portanto estaria subordinada a ela, enquanto 0 Studio-PhD parece utilizar um termo ultrapassado na era pós-estúdio. Além do mais, a insistência em utilizar 0 antiquado termo "belas-artes" está aberto a críticas.

\section{3}

Ver como referência: Annette W. Balkema e Henk Slager (org.), Artistic research, Amsterdam e New York: Rodopi, 2004; Katy McLeod e Lin Holdridge (org.), Thinking through art: critical reflections on emerging research, London: Routledge, 2005; Lesley Duxbury, Elizabeth M. Grierson e Dianne Waite (org.), Thinking through practice: art as research in the academy, Melbourne: Royal Melbourne Institute of Technology Publishing, 2007; Hazel Smith e Roger Dean, Practice-led research, research-led practice in the creative arts, Edinburgh: University of Edinburgh Press, 2009; Estelle Barrett e Barbara Bolt (org.), Practice as research: approaches to creative arts enquiry, London: I. B. Tauris, 2010. Veja também a edição especial de Working Papers in Art and Design, v. I, nov. 2000. 
James Elkins (org.), Artists with PhDs: on the new doctoral degree in studio art, New Academia Publishing, 2009.Ver também: Elkins, "Theoretical remarks on combined creative and scholarly PhD degrees in the visual arts", Journal of Aesthetic Education, v. 38, n. 4 , Winter 2004 , p. 22-31 Seguindo Charles Harrison (veja nota 7), Elkins argumentou que 0 termo "pesquisa" é inapropriado, tendo entrado no vocabulário de programas de doutorado como jargão administrativo. Em relação à "produção de conhecimento" como termo casado, o que é igualmente problemático, Elkins considera desnecessário e equivocado.

Veja 0 texto de Victor Burgin "Thoughts on research degrees in visual arts departments" em Elkins (org.), Artists with PhDs.

6

Elkins, "Brief conclusions" em Artists with PhDs, op. cit.

Christopher Frayling,"Research in art and design" em Royal College of Art Research Papers, v. I, n. I, 1993/4, p. I.Veja também seu recente Research degrees in art and design - why do people have problems with them?, apresentado como palestra no Royal College of Art em 2006.

8

Charles Harrison, "When management speaks", em Research and the artist: considering the role of the art school, organizado por Antonia Payne, publicado por Ruskin School of Drawing and Fine Art, Oxford, 2000 , p. 65. do que se toma por Teoria da Arte atualmente é um pouco melhor do que a baixa intensidade da História Social da Arte ou os exaustivos Estudos Culturais. Apesar disso, ninguém se iluda de que agora possam reproduzir-se como questões de conveniência institucional aquelas exóticas e altamente condicionadas circunstâncias sob as quais fazia sentido, há trinta anos, ser a Teoria da Arte seguida como uma forma crítica da prática artística. $^{10}$

Um bom número de anos após essas declarações, e cinco anos após a introdução do DPhil in Fine Art na Ruskin School of Drawing and Fine Art, a faculdade de belasartes da Universidade de Oxford, parece ser um bom momento para se refletir sobre nossa experiência e sobre os desafios e as aspirações que alimentam um programa que ainda se sente bastante novo, e que, felizmente, resiste a uma definição precisa. No processo de estabelecimento do programa, nós levamos em conta nossos pontos fortes, nossas idiossincrasias institucionais e também nossas restrições. A Ruskin é uma escola de arte pequena, sendo o tamanho de seu programa de graduação determinado, em certos aspectos, pelos requisitos do sistema tutorial de Oxford, que se baseia em tutorias um a um nas disciplinas. O "modelo Ruskin" é o de uma comunidade unida, e o programa DPhil refletiu a vontade de preservar isto. A maior vantagem ao nosso dispor foi que a Ruskin, ao contrário de muitas outras escolas de arte, faz parte de uma importante instituição de pesquisa. Isso também determinou que o programa seria incorporado a uma ampla estrutura preexistente de pesquisa e sujeito a uma série de normas acadêmicas estabelecidas, que permitiam margens limitadas de ajuste. Mas em termos de execução do programa, identificar e mobilizar as áreas pertinentes à experiência de pesquisa estabelecendo relações interdisciplinares e interdepartamentais tem sido um exercício fascinante. Desde a sua criação, o programa tem promovido modelos de troca de colaboração e de conhecimento junto a uma série de departamentos universitários, centros de pesquisa e museus. $\bigcirc$ inconfundível status do "artista em residência" foi invocado como um modelo que emprestou clareza a esse processo de suave infiltração de diferentes contextos institucionais pelos doutorandos da Ruskin. Com importantes pontos de referência, como o Pitt Rivers Museum, o Ashmolean Museum e a Modern Art Oxford, e centros de pesquisa que vão desde o Wellcome Trust até o Future of Humanity Institute ou o Centre for Environmental Studies, bem como a disponibilidade de pesquisas conduzidas dentro das disciplinas vizinhas das humanidades e através das ciências sociais e naturais (com a neurociência, a imagiologia médica e biotecnologia rapidamente estabelecendo-se como pontos focais de interesse para artistas), as possibilidades de intercâmbios interdisciplinares têm provado ser notavelmente abrangentes. $\mathrm{Na}$ 
prática, este processo tem também apresentado um desafio que é endêmico em todas as concepções da prática artística em termos de pesquisa interdisciplinar: Como se apresentam os resultados dessas excursões interdisciplinares ao serem re-importadas para o estúdio e como podem orientar a prática artística? O problema não é novo, pois é subjacente a todos os projetos "arte-ciência", os quais já se tornaram um componente estabelecido das práticas contemporâneas. Uma tentativa teórica de comprovar uma forte afinidade entre arte e ciência foi feita pelo ex-professor de história da arte da Universidade de Oxford, Martin Kemp. Baseando-se no conceito de "visualização", Kemp teorizou o processo pelo qual artistas e cientistas estruturam a realidade em termos de "intuições estruturais" compartilhadas." Ele as descreve como estruturas de processos intuitivos internos que são compartilhadas por artistas e cientistas, levando ao que ele chama de "atos de ver". Atos de ver (realidades fora de nós), Kemp argumenta, são estruturados em forma de

\section{[...] depósitos decorrentes da experiência perceptiva, critérios pré-estabelecidos de interpretação, ações novas e antigas de nomear e classificar, parâmetros físicos de nosso aparato sensorial e, sobretudo (e subjacente a tudo), estruturas profundas que operam a um nível pré ou subverbal. ${ }^{12}$}

Se isso for verdade, então oferece uma explicação de por que artistas e cientistas podem visualizar o mundo de maneiras análogas (e seus ensaios em Visualizations dão ampla evidência de surpreendentes sobreposições de imagem e intuição). Este consistente modelo pode de fato se aplicar a alguns casos, mas em termos mais gerais, tudo o que é sem dúvida razoável de se esperar da interação com a ciência é uma reação artística - uma reação em vez de uma interpretação.

A indefinição desta questão em aberto coloca um problema adicional para a conceituação da prática artística como pesquisa nestes casos interdisciplinares. Se estivéssemos de acordo em caracterizá-la, que tipo de pesquisa seria? Obviamente, não operaria em continuidade com a pesquisa científica básica.Tampouco envolveria ilustração e, desse modo, a propagação visual da ciência. Antes, a pesquisa estaria imbuída na prática artística, que por sua vez aspiraria ao modelo de um programa de pesquisa. Inevitavelmente, surge a necessidade de buscar precedentes. As experiências de Leonardo, cruzando fronteiras entre arte e ciência, oferecem um eminente paradigma - por serem drasticamente diferenciadas da obsessão acadêmica pela anatomia que constituiu a espinha dorsal da formação na Beaux-Arts por tanto tempo. Estudos da perspectiva e sua implementação visual propõem um precedente a mais, enquanto outro exemplo pode ser encontrado na noção de cubismo analítico como um programa de pesquisa. Mais recentemente, a experimentação de David Hockney

\section{9} Harrison, op. cit., p. 66.

\section{0}

lbidem

\section{II}

Martin Kemp, Seen/Unseen: Art, science and intuition from Leonardo to the Hubble telescope. Oxford University Press: Oxford 2006. E também , M. Kemp, Visualizations: The Nature Book of Art and Science, University of California Press: Berkeley, 200I.

12

Kemp, Visualizations, p. I. 
13

David Hockney, Secret knowledge: rediscovering the lost techniques of the old masters, London: Thames \& Hudson, 2006. com a câmara obscura e a aplicação direta de seus experimentos na pintura de retratos ${ }^{13}$ proporciona um exemplo contemporâneo de arte como pesquisa.

Outro desafio é apresentado pela natureza da escrita envolvida. A esse respeito, as opções principais, que têm sido exploradas por diferentes programas, parecem ser três: (a) escrita criativa, (b) reflexões sobre a prática, e (c) escrita acadêmica. As duas primeiras frequentemente representam o mesmo tipo de escrita envolvida em certo número de programas de mestrado em arte (MFA). Na Ruskin, ao menos na primeira etapa do desenvolvimento do programa DPhil, ambas as escritas eram estimuladas, mas, crucialmente, vistas como parte da produção do estúdio. Foi admitido que o trabalho de estúdio poderia envolver um elemento da "prática textual”, o qual podia ser bastante rico e cuja natureza estaria sob o título genérico de escrita criativa; e que a conceituação do projeto poderia recorrer a uma exploração escrita de vários aspectos da prática, a qual pode estender-se bem além do meramente explicativo e gerar peças de escrita altamente bem acabadas. Da perspectiva do programa Ruskin, nenhum destes pode ser canalizado para a tese. A definição exata da natureza da tese em si, de qualquer forma, permanece em aberto. Afora estipular que ela deveria se encaixar no projeto global de pesquisa, oferecendo alguma forma de contextualização para a prática (e demarcando sua extensão em até 40 mil palavras), não há especificações adicionais. A experiência tem mostrado que o conhecimento interno e distintivo das práticas artísticas que os artistas possuem pode gerar excelentes peças de crítica de arte. Isto indicaria que o contexto de práticas que compartilham objetivos comuns, ou de alguma forma operam em uma vertente similar a delas mesmas, é uma escolha óbvia em termos de tema de tese - embora esse contínuo e prolongado envolvimento com o trabalho de outros possa provar ser contraproducente de diversas formas. Confrontar a prática artística de uns com a de outros, tornar-se muito analítico sobre questões que se aproximem de nossa própria atividade criativa ou muito crítico em relação a vertentes de práticas que tenham afinidades semelhantes as nossas, intensificar uma percepção dos becos sem saída a serem evitados, todas estas são algumas das dificuldades potenciais que podem advir do amplo envolvimento teórico estreitamente relacionado às práticas artísticas e que compreensivelmente poderia inibir a prática, ao torná-la excessivamente auto-consciente.

Outro contexto óbvio é aquele das ideias. Nesse caso, a tese abordaria amplos contextos ideológicos e intelectuais, de relevância para a prática. Nessa qualidade, seria mais plausível interagir com disciplinas filosóficas, literárias ou da história, e com certas ciências sociais, como a etnografia, a antropologia, a geografia cultural, só para citar algumas. Um conjunto inteiro de difíceis questões metodológicas surge relacionado a esses padrões de interação. Para dentro dos limites dessas disciplinas, debates de temas que podem, inicialmente, vir ao encontro como de interesse geral, rapidamente se tornam altamente especializados. Como pode ser assegurado que uma escrita 
rigorosa é produzida, quando se interage com uma disciplina na qual não se teve um ensino formal? Que padrões devem ser aplicados? E como é o tema a ser abordado metodologicamente? O princípio norteador é aquele determinado empiricamente e numa base de caso-a-caso, com supervisão especializada desempenhando um papel chave em circum-navegar alguns dos mais difíceis problemas de se resolver. Em todos os casos, está-se condicionado pelos padrões críticos aplicáveis ao discurso existente: não é possível envolver, por exemplo, filosofia analítica sem utilizar argumentação sustentada, ou abordar um problema formulado dentro da história social da arte sem recorrer a esse enquadramento metodológico específico, se somente para transcendê-lo.

A objeção que prevalece para todos os casos acima é a de que o doutorado em arte recai sobre - e inevitavelmente leva a - uma excessiva academização da prática artística. Há um elemento de verdade nisso. Mas ainda que aspectos do projeto possam ser dominados pelo trabalho acadêmico, a prática em si deveria construir fortes defesas contra qualquer potencial academização. $O$ perigo iminente é o da deterioração de algumas formas de ilustração de ideias teóricas; e, também, da perda de interesse intrínseco, fundamentalmente da perda de autonomia. Há um delicado equilíbrio a ser alcançado: a prática é complementada pelo componente "acadêmico" (o termo "acadêmico" é usado aqui na falta de um que funcione melhor já que "teoria” impõe uma dicotomia entre teoria e prática, que persiste profundamente problemática), ao mesmo tempo que enfaticamente afirma sua independência.

A longa e diversificada tradição dos escritos de artistas proporciona um interessante campo de comparações. Uma série de proeminentes exemplos vem à mente: Robert Smithson, Daniel Buren, Donald Judd, Robert Morris, Joseph Kosuth, Art \& Language, Gerhard Richter e Jeff Wall, para citar alguns, todos se engajaram a uma intensa prática textual. ${ }^{14}$ Jeff Wall, largamente descrito como "um artista que escreve" (uma descrição que soa um tanto estranha), tem sido indagado sobre seus escritos em uma série de entrevistas. ${ }^{15}$ A visão que tem de sua própria prática de escrita é particularmente interessante: ele a descreve como uma atividade paralela - e é esse modo de existir em paralelo, como parte de um todo orgânico, que colabora precisamente para uma compreensão pelo menos do componente escrito da tese de doutorado em arte. Curiosamente, na maioria desses casos, os escritos envolvem ideias de dentro do amplo contexto das práticas artísticas e não se entregam a reflexões subjetivas ou a criativas modalidades literárias. Ao mesmo tempo, sua dimensão é a de um ensaio em vez de uma extensa tese, e assim, considerá-los como precedentes empresta suporte aos aspectos escritos dos projetos de doutorado em algumas circunstâncias, mas talvez não em outras. A melhor recomendação é manter questões em aberto, conservar tanto quanto possível flexibilidade na definição da tese, ter em mente que para todo projeto artístico há numerosas opções de tópicos
14

Ver Robert Smithson: the collected writings, Jack Flam (org.), University of California Press, 1996; Robert Morris, Continuous project altered daily: the complete writings of Robert Morris, MIT Press, 1991; Joseph Kosuth, "Art after philosophy" (1969) em Art after philosophy and after, MIT Press, Cambridge, 1993; Gerhard Richter, The daily practice of painting, Hans-Ulrich Obrist (org.), London: Thames \& Hudson, 1995; Jeff Wall, Selected essays and interviews, New York: The Museum of Modern Art, 2007.

15

Ver, por exemplo, "Writing on art: interview between Jeff Wall and Jean-Francois Chevrier", em Jeff Wall: selected essays and interviews. 
16

Uma interessante e idiossincrática genealogia da transição para 0 conceito genérico da arte pode ser encontrada em Thierry de Duve, Kant after Duchamp, Cambridge: MIT Press, 1996.

17

Daniel Buren, "The function of the studio", October, v. 10, Autumn, 1979, p. 51-58. De uma coleção de abordagens diversas no estúdio do artista, ver Mary Jane Jacob e Michelle Grabner (org.), The studio reader: on the space of artists, Chicago e London: University of Chicago Press, 2010. que podem ser conduzidos de modo produtivo, e que no melhor dos casos, estes poderão até mesmo estimular a prática artística, ainda que, decididamente, os padrões tendam a ser implícitos e complexos.

Em todos os casos, seria ideal que programas de doutorado proporcionassem ricos contextos intelectuais, importando tantas ideias quanto possível de seu ambiente de pesquisa circundante. Uma infra-estrutura básica de seminários de pesquisa realizada ou em andamento, palestras e conversas de artistas convidados, assim como a criação de numerosas redes de pesquisa e artísticas e parcerias apoiam esse esforço. Desde as fases iniciais de elaboração do programa de doutorado na Ruskin, nós buscamos manter o potencial de sua mais importante característica: o fato de que abrange duas vertentes. A Dphil dirigida com base prática coexiste com a Dphil teórica, oficialmente conhecida como "Dphil de tese única" [DPhil by thesis only] - a nomenclatura sendo indicativa das dificuldades surgidas em se tentar definir sua natureza, especialmente quando envolve o termo "teoria". Ambas as vertentes são estritamente partes do mesmo contexto e infraestrutura acadêmica. O DPhil teórico (para reportar a esse termo abreviado de modo a distingui-lo de doutorado com base prática) é, certamente, um território acadêmico familiar. A atração em seguir pesquisa teórica de doutorado em uma escola de arte, diferentemente de um departamento universitário especializado, vem do alto grau de especialização em arte contemporânea, mas também do fato de que o etos interdisciplinar da escola de arte contemporânea (onde o conceito genérico de arte tem, em grande medida, superado as mais antigas divisões entre categorias artísticas ${ }^{16}$ ) oferece um ambiente de incentivo à pesquisa menos afetado pela preocupação com fronteiras entre disciplinas. Pesquisadores de doutorado que seguem esse caminho são frequentemente motivados por um desejo de interdisciplinaridade que considera as limitações de ter que operar dentro de um território claramente delimitado por uma disciplina acadêmica específica como pouco construtiva. Isso não significa que as questões metodológicas no âmbito da investigação interdisciplinar sejam evitadas. Ao contrário, elas são confrontadas desde o início e desde então continuamente, gerando o andamento de um debate de meta-nível, ou de segunda ordem, que é particularmente produtivo. Além do mais, as duas vertentes participam em um diálogo de aprimoramento recíproco e fortalecimento mútuo: os artistas submetem seus trabalhos e ideias à análise crítica não só de outros artistas como também de teóricos que as abordam de diversos e, ocasionalmente, inesperados pontos de vista; e os teóricos, por sua vez, "não estão autorizados" a destacarem-se demais, mas terem suas idéias submetidas a por vezes implacável prova real do estúdio. O resultado, em ambos os casos, é altamente construtivo - e ainda oferece potencial para gerar atividade de pesquisa conjunta e de projetos curatoriais colaborativos.

A referência indireta à crítica de Daniel Buren às estruturas institucionais em "The Function of the Studio", ${ }^{17}$ em meu título, aponta para o reposicionamento 
idiossincrático do estúdio como um estudo na era dos "artistas com doutorado". Descrevendo o estúdio como uma torre de marfim, Buren defende que ele está conectado ao museu e à galeria "para compor a fundação do mesmo edifício e do mesmo sistema". ${ }^{18}$ Em sua reinvenção como um estudo, o estúdio não passa de uma torre de marfim. Embora isso possa ser submetido a novas vertentes da crítica institucional, em outro plano é simplesmente algo para ser observado. $O$ doutorado em arte representa uma variada matriz de possibilidades para artistas interessados em um compromisso continuado com a vida acadêmica. Um dos maiores desafios que colocados reside em acertar o equilíbrio certo entre envolvimento e distanciamento, em manter a vitalidade da arte e não ficar confortável demais dentro da torre de marfim, uma vez que, assim como outras formas de atividade intelectual, a arte padece quando leva a si mesma muito a sério.

18

Buren, "The Function of the Studio", p. 51.

\section{KATERINA REED-TSOCHA}

Coordenadora do Programa de Pós-Graduação da Ruskin School of Drawing and Fine Art, University of Oxford, de 2007 a 2012, foi Research Fellow no Trinity College Oxford. Lecionou no Christ Church e no St Hilda's College, Oxford, no Departamento de História da Arte de Oxford e na Open University. Como Diretora de Pós-Graduação na Ruskin School, tem sido responsável pelo desenvolvimento do recentemente criado doutorado em belas-artes e supervisiona doutorandos que exercem simultaneamente pesquisa de base prática e teórica; também leciona história e teoria da arte na graduação. Sua pesquisa é interdisciplinar e pode ser situada na intersecçãa entre a história da arte e a filosofia. Suas publicações recentes incluem: "Study the masters? On the ambivalent status of art history within the contemporary art school", em The concept of the master, organizado por Matthew Potter, Ashgate, 2012 (no prelo); "Vasarely's multiples: their artistic context and long-term philosophical significance", em The challenge of the object / Die Herausforderung des Objekts, Congress Proceedings, vol. 2, organizado por G. Ulrich Grossmann e Petra Krutisch, Nuremberg, 2013 (no prelo); “Collective action and the reciprocity of friendship”, em Collaborating Now, organizado por Sondra Bacharach, Jeremy Booth e Siv B. Fjærestad, para 2013; "Mortal vocabularies vs. immortal propositions: Richard Rorty and the conversation of mankind", Culture and Dialogue 2 (set. 20II); e os livros Expressionism: a very short introduction, Oxford University Press, e The myth of the unique piece, previstos para 2013, e The excess of theory (em andamento). 
